\title{
Hierarchical decomposition of datasets on irregular surface meshes
}

\author{
Georges-Pierre Bonneau \\ Alexandre Gerussi \\ LMC-CNRS, University Joseph Fourier \\ BP 53, 38041 Grenoble Cedex 9, France \\ E-mail: [bonneau, Alexandre.Gerussi] @imag.fr
}

\begin{abstract}
In this paper we introduce multiresolution analysis (MRA) algorithms intended to be used in scientific visualization, and based on a non-nested set of approximating spaces. The need for non nested spaces arises from the fact that the required scaling functions do not fulfill any refinement equation. Therefore we introduce in the first part the concept of approximated refinement equation, that allows to generalize the filter bank and exact reconstruction algorithms. The second part shows how this concept enables to define a MRA scheme for piecewise constant data defined on an arbitrary planar or spherical triangularmesh. The ability to deal with arbitrary triangular meshes, without subdivision connectivity, can be achieved only through the use of non nested approximating spaces, as introduced in the first part.
\end{abstract}

\section{Introduction}

Hierarchical decomposition of datasets based on wavelet theory is a growing subject of research in computer graphics and scientific visualization. Wavelet theory is based on the knowledge of a sequence of functional spaces in which the data is successively approximated. Unfortunately, this sequence has to be nested. This explains why wavelet theory can't be applied to the multiresolution analysis of datasets defined on irregular meshes, since such meshes cannot be reach by a subdivision process starting on a coarser mesh.

Recently ([1]) we have introduced a theoretical framework that enables to deal with a non-nested sequence of functional spaces. The link between successive non-nested spaces is given through the definition of intermediate spaces. We will show how this framework can be applied to the multiresolution analysis of datasets defined on irregular surface meshes. We will combine our results with a well-known hierarchical structure- the hierarchical Delaunay triangulation- in order to obtain successive approximations of the original dataset on simplified meshes. We will show several examples that demonstrate the stability of our decomposi- tion/reconstruction algorithms, as well as the validity of our choice for the wavelet functions. We will explain how we can use the wavelet coefficients in order to optimize the hierarchical decomposition of the dataset.

\section{Multiresolution Analysis with non-nested approximating spaces}

We assume that the reader is familiar with the basic concepts of multiresolution analysis. An introduction on this topic can be found in [6]. In this section, a general framework for constructing multiresolution analysis schemes based on a non nested sequence of approximating spaces is given. All functional spaces throughout the paper are supposed to have finite dimensions.

Suppose you are given a sequence of functional spaces $V_{n}$ that satisfies the following condition:

$$
V_{n} \text { is isomorph to a subspace of } V_{n+1} \text {. }
$$

This condition means that the sequence of approximating spaces is "growing": it may be rewritten as $\operatorname{dim} V_{n} \leq \operatorname{dim} V_{n+1}$.

\section{Approximated refinement equation}

Let $\left(\varphi_{j}^{n}\right)$ denote the scaling functions (i.e. $\left(\varphi_{j}^{n}\right)$ is a basis of $V_{n}$ ). If the usual nested condition $V_{n} \subset V_{n+1}$ would be satisfied, the link between two successive levels of resolution would be provided by a so-called refinement equation, which expresses each scaling function $\varphi_{j}^{n}$ as a linear combination of the finer scaling functions $\left(\varphi_{i}^{n+1}\right)$. In our setting, condition (1) implies that there exists functions $\left(\tilde{\varphi}_{j}^{n}\right)$ which form a basis of a space $\tilde{V}_{n}$, isomorph to $V_{n}$, and included in $V_{n+1}$. And the link between level $n$ and $n+1$ is now provided through a two step process: First apply the isomorphism, i.e. replace the scaling functions $\left(\varphi_{j}^{n}\right)$ by the intermediate functions $\left(\tilde{\varphi}_{j}^{n}\right)$, and then apply the refinement equation which expresses $\tilde{\varphi}_{j}^{n}$ as a linear combination of the finer scaling functions $\left(\varphi_{i}^{n+1}\right)$ : 


$$
\varphi_{j}^{n} \leftrightarrow \tilde{\varphi}_{j}^{n}=\sum_{i} p_{i j}^{n} \varphi_{i}^{n+1}
$$

We refer to (2) as the approximated refinement equation $\mathrm{A}$ crucial point of our method is the choice of the intermediate scaling functions $\left(\tilde{\varphi}_{j}^{n}\right)$. This choice will be precised later.

\section{Wavelet functions and filter bank}

Once the intermediate functions, and thus the intermediate spaces $\tilde{V}_{n}$ are chosen, we choose the detail space $W_{n}$ as a complementary of the intermediate space $\tilde{V}_{n}$ in the finer space $V_{n+1}$ :

$$
V_{n+1}=\tilde{V}_{n} \oplus W_{n}
$$

The wavelet functions $\left(\psi_{j}^{n}\right)$ are basis functions of the space $W_{n}$. Thus there exist coefficients $\left(q_{i j}^{n}\right)$ such that

$$
\psi_{j}^{n}=\sum_{i} q_{i j}^{n} \varphi_{i}^{n+1},
$$

and coefficients $\left(a_{i j}^{n}\right),\left(b_{i j}^{n}\right)$ such that

$$
\varphi_{j}^{n+1}=\sum_{i} a_{i j}^{n} \tilde{\varphi}_{i}^{n}+\sum_{i} b_{i j}^{n} \psi_{i}^{n}
$$

Let $A, B, P, Q$ denote the matrices with coefficients $\left(a_{i j}^{n}\right),\left(b_{i j}^{n}\right),\left(p_{i j}^{n}\right),\left(q_{i j}^{n}\right)$. Given an approximation $f_{n+1}=\sum_{i} x_{i}^{n+1} \varphi_{i}^{n+1}$ in $V_{n+1}$, the next coarser approximation $f_{n}=\sum_{i} x_{i}^{n} \varphi_{i}^{n}$ and the details $g_{n}=\sum_{i} y_{i}^{n} \psi_{i}^{n}$ are computed by

$$
\left(x_{i}^{n}\right)=A\left(x_{i}^{n+1}\right) \quad\left(y_{i}^{n}\right)=B\left(x_{i}^{n+1}\right) .
$$

The inverse transformation is given by

$$
\left(x_{i}^{n+1}\right)=P\left(x_{i}^{n}\right)+Q\left(y_{i}^{n}\right) .
$$

The analysis and reconstruction formulas (3) and (4) are the same as if the nested condition $V_{n} \subset V_{n+1}$ would be satisfied, but they are related now to an approximation in two steps:

$$
\begin{gathered}
f_{n+1} \stackrel{\text { app. }}{\longrightarrow} \tilde{f}_{n}=\sum_{i} x_{i}^{n} \tilde{\varphi}_{i}^{n} \stackrel{\text { iso. }}{\leftrightarrow} f_{n}=\sum_{i} x_{i}^{n} \varphi_{i}^{n} \\
g_{n}
\end{gathered}
$$

\section{Choice of the analysis and synthesis matrices}

The complete choice for the analysis and synthesis matrices depends on the application, and thus will be made in the next section. Nevertheless, some conditions that these matrices have to fullfill in order to ensure best-approximation and orthogonality properties can be precised now.
Let $G_{n}$ denote the Gram-schmidt matrix of the basis $\varphi_{i}^{n}$ (i.e. the matrix whose elements are $\left\langle\varphi_{i}^{n}, \varphi_{j}^{n}>\right.$ ). If we want $f_{n}$, the approximation at resolution $n$, to be the best $L_{2}$ approximation of $f_{n+1}$, then it is easy to show that the analysis matrix $A$ must satisfy the following condition:

$$
A=G_{n}^{-1}\left(<\varphi_{i}^{n}, \varphi_{j}^{n+1}>\right) .
$$

Once $A$ is computed from (5), the matrix $B$ can be chosen orthogonal to $A$, and orthonormal, with respect to the scalar product in the dual scaling function basis of $V_{n+1}$ :

$$
\begin{aligned}
A G_{n+1}^{-1} B^{T} & =0 \\
B G_{n+1}^{-1} B^{T} & =I .
\end{aligned}
$$

$P$ and $Q$ are then computed from $A$ and $B$ in order to ensure the exact reconstruction property:

$$
(P Q)=\left(\begin{array}{c}
A \\
B
\end{array}\right)^{-1} .
$$

Together, conditions (5), (6), (7), and (8) ensure that the approximation at resolution $n f_{n}$ is the best $L_{2}$ approximation of both $f_{n+1}$ and the intermediate approximation $\tilde{f}_{n}$.

\section{Application to piecewise constant data sets on irregular triangulations}

In this section, the theoretical results of section 2 are applied to the multiresolution analysis of piecewise constant data sets on irregular planar or spherical triangular meshes.

Previous works on wavelet methods for data sets defined on triangular meshes are restricted to regular meshes obtained by recursively spliting in 4 triangles each triangle of a coarse base mesh ([5], [4], [3]) (see fig. 1). This recursive process is associated to a simple hierarchy, that has the structure of a forest of quadtrees. For such meshes, it is possible to construct a nested sequence of approximation spaces.

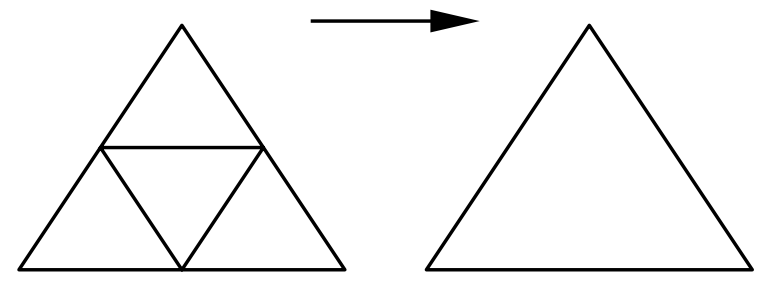

Figure 1. 4-to-1 split

When dealing with irregular triangulations, it is not possible to associate a simple tree structure to the data set. Other 
possible hierarchies may be constructed by applying edge contraction, triangle contraction or vertex removal operation on the triangular mesh. These hierarchies cannot be represented by tree structures, and no sequence of nested approximation spaces can be associated. We have chosen to use vertex removal operations (see fig. 2), and more specifically we have applied previous works from Kirkpatrick ([2]) to build a so-called hierarchical Delaunay structure. This is explained in subsection 3.1.

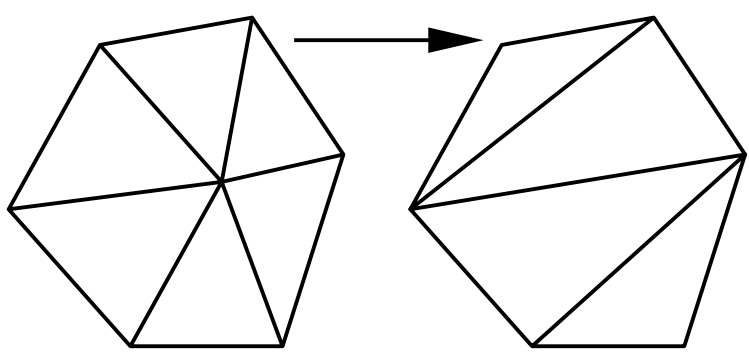

Figure 2. Point removal

Once the hierarchy has been computed, we have applied the results of section 2 locally to each star-shaped polygon created by the vertex removal operations, in order to compute new face values on the new triangles, as well as detail values that measure the distance error between the original data set and the new simplified one (as illustrated in fig. 3). This will be explained in subsection 3.2.

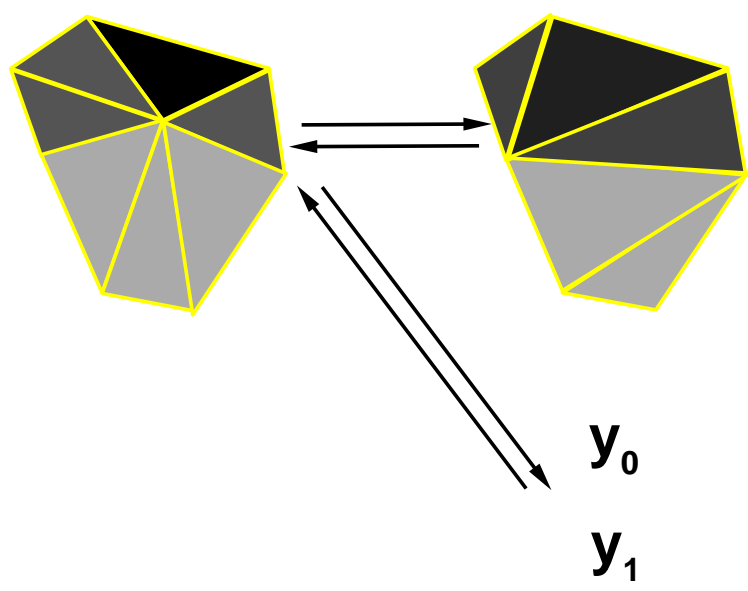

Figure 3. Computation of new face values and detail coefficients

Eventually we show in subsection 3.3 how the local analysis and synthesis described in 3.2 combine with the hierarchy introduced in 3.1 in order to yield global analysis and synthesis algorithms for piecewise constant data sets defined on irregular planar or spherical triangular meshes.

\subsection{Hierarchical Delaunay Triangulation}

Starting from a surface triangulation, the construction of a hierarchical Delaunay structure, as introduced in 1983 by Kirkpatrick ([2]), can be sketched as follows:

While (\# selected vertices $>0$ )

- select a maximal set of independent (non-adjacent) vertices

- remove each of the selected vertex and their adjacent edges $\Rightarrow$ creation of blocs,

- retriangulate the blocs according to a Delaunay criteria,

- update the links between the levels.

Although it was introduced for planar triangulations, this algorithm can be applied to spherical triangulations as well. It can be implemented with several data structures. Depending on the data structure, the links can join either faces, vertices or blocs between different levels. Figure 4 shows an example of such a hierarchy. The original and simplified triangulations are shown on the left. Empty circles point to vertices that are removed to reach the next level. The middle part shows the corresponding hierarchies over the faces. Black circles denote triangles, and links join triangles that have non-empty intersections. Rounded-rectangles group triangles belonging to the same bloc. The right part of figure 4 shows these blocs, and the links between intersecting blocs. Each bloc has two triangulations: triangles before the vertex removal (drawn solid) are called child triangles of the bloc, and triangles after the vertex removal (in dashed) are called parent triangles.

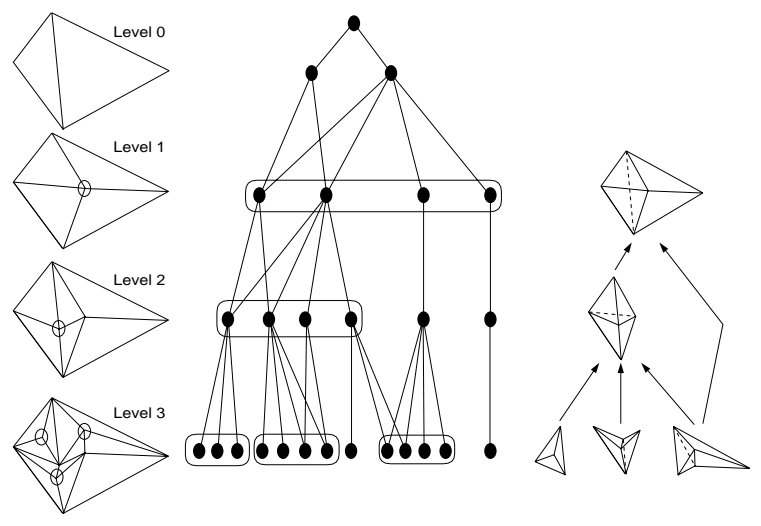

Figure 4. Hierarchical Delaunay triangulation

\subsection{Local analysis and synthesis}

The hierarchy construction described in the previous subsection is based on the retriangulation of star-shaped polygons on the surface mesh. In this subsection, we apply the 
results of section 2, locally to these star-shaped polygons. This leads to a local analysis and synthesis scheme of the data set.

Let $p$ and $q$ be respectively the number of triangles before and after the vertex removal. Euler formula implies $p=q+2$. Let $\left(T_{i}^{f}\right)_{i=1 \ldots p}$ denote the triangles before the vertex removal, and $\left(T_{i}^{c}\right)_{i=1 \ldots q}$ the triangles after the vertex removal (the superscript $f$ stands for "fine" and $c$ for "coarse"). Since we are dealing with piecewise constant data sets, let $V^{f}$ denotes the space of piecewise constant functions on the triangles $T_{i}^{f}$ and $V^{c}$ on the triangles $T_{i}^{c}$, then:

$$
\operatorname{dim}\left(V^{f}\right)=q<\operatorname{dim}\left(V^{c}\right)=p=q+2 .
$$

The basis function $\varphi_{i}^{f}$ in $V^{f}$ equals 1 on the triangle $T_{i}^{f}$ and 0 elsewhere (analogously for the basis functions in $V^{c}$ ):

$$
\begin{gathered}
\varphi_{i}^{f}=\chi_{T_{i}^{f}} \\
\varphi_{i}^{c}=\chi_{T_{i}^{c}} .
\end{gathered}
$$

The Gram-schmidt matrices $G^{f}$ and $G^{c}$ in $V^{f}$ and $V^{c}$ are the following diagonal matrices:

$$
\begin{aligned}
G^{f} & =\operatorname{diag}\left(\operatorname{area}\left(T_{i}^{f}\right)^{2}\right) \\
G^{c} & =\operatorname{diag}\left(\operatorname{area}\left(T_{i}^{c}\right)^{2}\right) .
\end{aligned}
$$

Therefore, equation (5) leads to the following local analysis matrix $A$ :

$$
A=\left(\frac{\operatorname{area}\left(T_{i}^{c} \cap T_{j}^{f}\right)}{\operatorname{area}\left(T_{i}^{c}\right)}\right)
$$

The computation of the analysis matrix $B$ is done by first fixing a sub-matrix of $B$ to be the identity, second solving the homogeneous linear system (6), and third performing a Gram-schmidt orthonormalization process on the rows the solution, with respect to the scalar product of matrix $\left(G^{f}\right)^{-1}$. The Gram-schmidt orthonormalization process ensures that the orthonormality condition (7) is fullfilled by the matrix $B$.

Figure 5. illustrates the application of the analysis scheme on one example: the top part shows the input function to the left (in the finer space $V^{f}$ ), the intermediate approximation in the middle (in the intermediate space $\tilde{V}^{c}$ ), and the coarser approximation to the right (in the coarser space $V^{c}$ ). The bottom part of figure 5 shows the two wavelet components (in the detail space $W^{c}$ ).

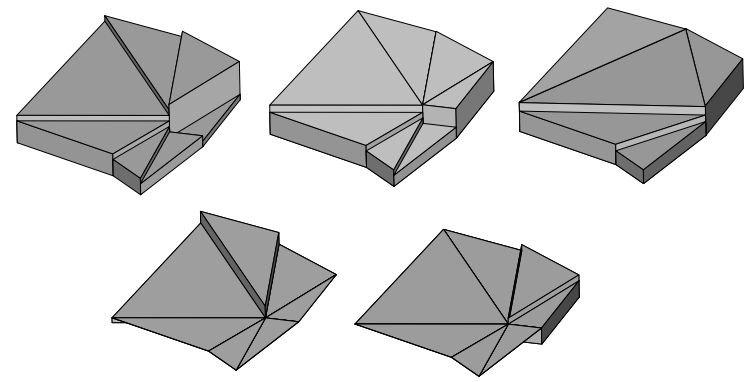

Figure 5. Local analysis and synthesis: finer, intermediate and coarser approximations on top; wavelet components on bottom.

\subsection{Global analysis and synthesis}

The global analysis of a piecewise constant data set defined on an irregular planar or spherical triangulation results from the application of the local analysis formulas (1) and (2), with the analysis matrices described in subsection 3.2, in each bloc of the hierarchical Delaunay structure introduced in subsection 3.1, starting from the finest level, and going up to the coarsest level.

After the whole analysis, each removed vertex has been assigned two wavelet coefficients. Partial reconstructions of the data set, using only wavelet coefficients of high magnitude can then be performed. This can be done by selecting a sub-hierarchy of the hierarchical Delaunay structure that contains these wavelet coefficients, and applying the reconstruction formula (4) in each bloc of this sub-hierarchy, starting from the finest level, and going up to the coarsest level. Of course, the insertion of all wavelet coefficients yields exactly the original data set.

Figure 6 shows an example on a spherical data set with $1.3 \mathrm{M}$ faces. The data set is defined on a regular triangulation (4-to-1 split with 8 levels of subdivision starting on a icosahedron). The data value on each face derives from the ETOPO5 ${ }^{1}$ data set (which consists of $2160 \times 4320$ samples on a uniform grid). Figure 6 shows a partial reconstruction using the highest wavelet coefficients, with 150000 triangular faces (out of $1.3 \mathrm{M}$ ). The selection of the highest wavelet coefficients clearly results in the insertion of more vertices where the data set has sharp variations, and less vertices elsewhere, thereby validating our choice for the analysis and synthesis matrices.

\footnotetext{
${ }^{1}$ Available via anonymous ftp at ftp: / ftp.ngdc.noaa.gov/Solid Earth/Topo
} 

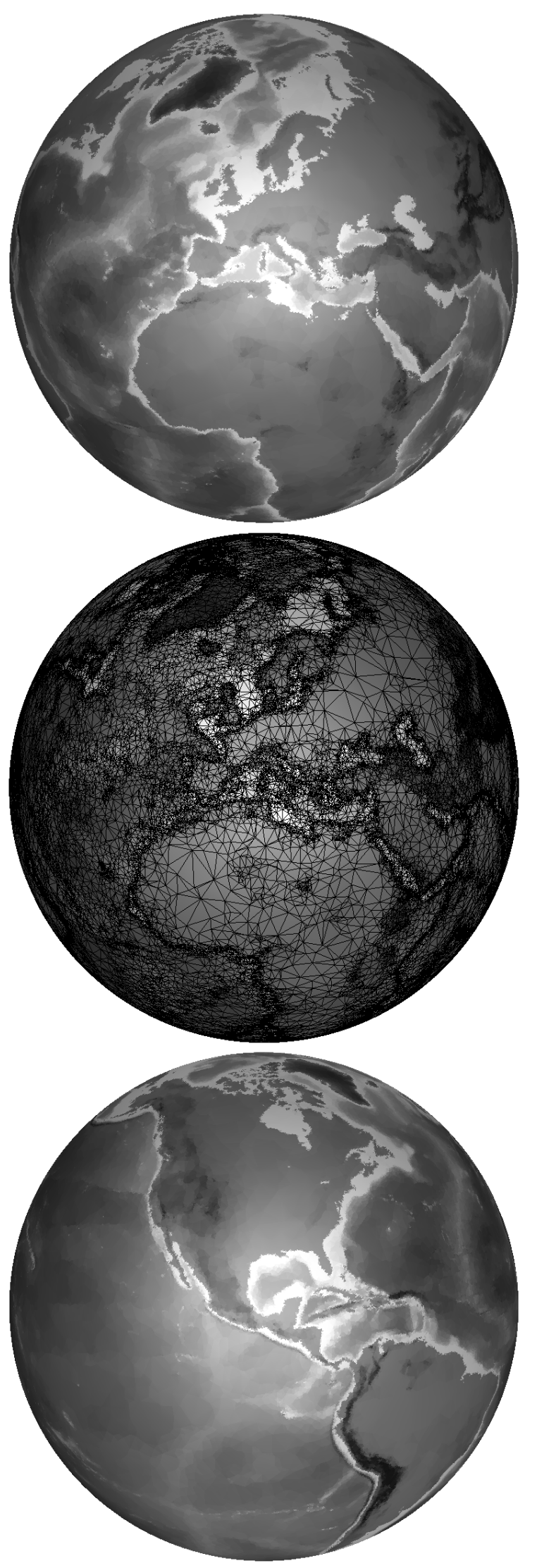

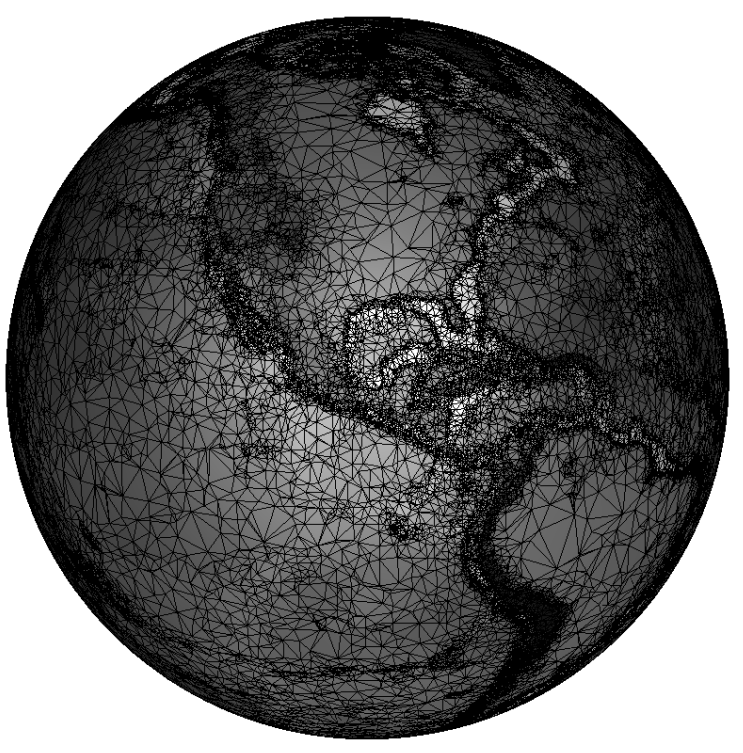

Figure 6. Partial reconstructions with 150000 faces (out of 1.3M)

\section{References}

[1] G. Bonneau, S. Hahmann, and G. M. Nielson. BLaCWavelets: A multiresolution analysis with non-nested spaces. In IEEE Visualization '96, pages 43-48. IEEE, Oct. 1996.

[2] D. Kirkpatrick. Optimal search in planar subdivisions. SIAM Journal on Computing, 12(1):28-35, Feb. 1983.

[3] M. Lounsbery, T. D. DeRose, and J. Warren. Multiresolution analysis for surfaces of arbitrary topological type. ACM Transactions on Graphics, 16(1):34-73, Jan. 1997.

[4] G. M. Nielson, I.-H. Jung, and J. Sung. Haar-wavelets over triangular domains with applications to multiresolution models for flow over a sphere. In IEEE Visualization '97, pages 143-150. IEEE, Nov. 1997.

[5] P. Schröder and W. Sweldens. Spherical wavelets: Efficiently representing functions on the sphere. In SIGGRAPH 95 Conference Proceedings, pages 161-172. ACM SIGGRAPH, Aug. 1995.

[6] E. J. Sollnitz, T. D. DeRose, and D. H. Salesin. Wavelets for Computer Graphics: Theory and Applications. Morgan Kaufmann Publishers, 1996. 